\title{
Ferrite resonance cones
}

\author{
P. M. Bellan \\ California Institute of Technology, Pasadena, California 91125
}

(Received 6 December 1982; accepted for publication 4 March 1983)

Resonance cones, a phenomenon which has been observed in electrostatic plasma waves, should also exist in magnetostatic waves propagating in ferrites. Resonance cones, the spatial characteristics of the hyperbolic partial differential describing both plasma and ferrite waves, are aligned along the group velocity trajectory and are equivalent to the superposition of an infinity of normal modes. The existence of resonance cones has been overlooked in standard normal mode analyses of ferrite waves, where typically, only one mode is assumed to exist at a time.

PACS numbers: $75.80 .+\mathrm{q}$

There is a formal equivalence ${ }^{1}$ between the equations describing electrostatic oscillations in plasmas and magnetostatic oscillations in ferrites, and so there is a correspondence between many of the modes in the two media. I would like to point out here the existence of a mode which has been studied in great detail in plasmas, ${ }^{2-4}$ but, to the best of my knowledge, has not been previously noted in ferrites.

This mode, called the resonance cone is not really a mode at all, but is rather a superposition of modes, in the sense that a Dirac delta function is a superposition of Fourier modes $\sim \exp (i k x)$. The derivation presented here will be similar to Fisher and Gould's ${ }^{2}$ derivation for plasma resonance cones.

The magnetostatic wave equations for a ferrite are ${ }^{5}$

$$
\begin{aligned}
& \mathbf{H}=-\nabla \varphi, \\
& \nabla \cdot \mathbf{B}=0, \\
& \mathbf{B}=\boldsymbol{\mu} \cdot \mathbf{H},
\end{aligned}
$$

and

$$
\nabla \cdot(\boldsymbol{\mu} \cdot \mathbf{H})=0,
$$

where the permeability tensor is

$$
\boldsymbol{\mu}=\mu_{0}\left[\begin{array}{ccc}
\mu_{1} & -i \mu_{2} & 0 \\
i \mu_{2} & \mu_{1} & 0 \\
0 & 0 & 1
\end{array}\right] \text {, }
$$

and

$$
\begin{aligned}
& \mu_{1}=1+\frac{G}{1-F^{2}}, \quad \mu_{2}=\frac{G F}{1-F^{2}}, \\
& F=\omega / \gamma H_{i}, \quad G=4 \pi M_{s} / H_{i} .
\end{aligned}
$$

Here, $\gamma$ is the gyromagnetic ratio, $4 \pi M_{s}$ the saturation magnetization, $H_{i}$ the internal (taking into account demagnetization factors) dc magnetic field applied in the $z$ direction, and $F$ the normalized frequency. Losses are neglected. By performing appropriate Fourier or Fourier/Bessel transformations, Eqs. (1)-(6) have been solved in Refs. 5-11 to find normal modes in various geometries (e.g., sphere, ellipsoid, disc, rod, bar, slab, strip, post) and much emphasis has been given to the correct matching of the boundary conditions at the interface between the ferrite and the external medium (typically vacuum or a conductor).

However, as has been shown for plasmas, when the medium is infinite in extent it is possible, without resorting to
Fourier modes, to find a general solution of Eq. (4) in terms of the characteristics of a hyperbolic partial differential equation. In the following, resonance cone solutions of Eq. (4) will be derived in both Cartesian and cylindrical coordinates. The reason for repeating the derivation in two coordinate systems is that each sheds light on different, but complementary, aspects of resonance cones.

In Cartesian coordinates and for homogeneous media, Eq. (4) has the form

$$
\mu_{1}\left[\frac{\partial^{2} \varphi}{\partial x^{2}}+\frac{\partial^{2} \varphi}{\partial y^{2}}\right]+\frac{\partial^{2} \varphi}{\partial z^{2}}=0 .
$$

For an antenna lying in the $y z$ plane and having no dependence on $y$ (i.e., $\partial / \partial y=0$ ), and for the parameter regime where $\mu_{1}<0$, the solution of Eq. (7) is of the form

$$
\varphi(x, z)=f\left(x \pm \sqrt{-\mu_{1}} z\right),
$$

where $f$ is determined by the antenna's dependence on $z$. In other words, the solutions propagate along the characteristics $x \pm \sqrt{-\mu_{1}} z=$ const. of the hyperbolic partial differential equation. The potential on a particular characteristic is determined by the magnetostatic potential of the portion of the antenna which intersects the characteristic.

The angle the characteristic subtends with respect to the $z$ axis is called the resonance cone angle $\theta_{c}$, and is given by

$$
\tan \theta_{c}=\frac{d x}{d z}= \pm \sqrt{-\mu_{1}}
$$

It is important to realize that $\theta_{c}$ is not the same as $\theta_{k}$, the angle the wave vector $\mathbf{k}$ of a plane wave $\sim \exp (i \mathbf{k} \cdot \mathbf{x})$ subtends with respect to the $z$ axis. This may be seen by Fourier analyzing Eq. (7) in space to give the dispersion relation

$$
D(\mathbf{k}, \omega)=k_{1}^{2} \mu_{1}+k_{z}^{2}=0,
$$

where $k_{\perp}=\left(k_{x}^{2}+k_{y}^{2}\right)^{1 / 2}=k \sin \theta_{k}$ and $k_{z}=k \cos \theta_{k}$ are, respectively, the wave vector components perpendicular and parallel to the dc magnetic field. From Eq. (10)

$$
\tan \theta_{k}= \pm \frac{1}{\sqrt{-\mu_{1}}}
$$

thus $\theta_{c}$ and $\theta_{k}$ are orthogonal. ${ }^{2}$

This surprising result is a manifestation of the fact that the group and phase velocities are orthogonal. This assertion may be proved by calculating the group velocity from Eq. (10): 
$\mathbf{v}_{g r}=\frac{\partial \omega}{\partial \mathbf{k}}=-\frac{\partial D}{\partial \mathbf{k}} / \frac{\partial D}{\partial \omega}=-2\left(\mathbf{k}_{1} \mu_{1}+k_{z} \hat{\mathbf{z}}\right) / \frac{\partial D}{\partial \omega}$

The phase velocity is aligned along $\mathbf{k}$; i.e., $\mathbf{v}_{p h}=\omega \mathbf{k} / k^{2}$, so

$$
\mathbf{v}_{g r} \cdot \mathbf{v}_{p h}=-2\left(\mathbf{k}_{\perp} \mu_{1}+k_{z} \hat{\mathbf{z}}\right) \cdot \mathbf{k} \omega / k^{2} \frac{\partial D}{\partial \omega}=0,
$$

using Eq. (10). This orthogonality is a general property of dispersion relations which [like Eq. (10)] depend only on $\theta_{k}$ and not on the magnitude of $\mathbf{k}$. This is easily seen by expressing the operator $\partial / \partial \mathbf{k}$ in cylindrical coordinates $\left(k, \theta_{k}, z\right)$. Since $D$ does not depend on $k$ (nor, for homogeneous media, on $z), \partial D / \partial \mathbf{k}=\hat{\boldsymbol{\theta}}_{k} k^{-1} \partial D / \partial \boldsymbol{\theta}_{k}$, which has no component in the $\mathbf{k}$ direction.

Since the group velocity and $\theta_{c}$ are both orthogonal to $\theta_{k}$, the group velocity angle must be $\theta_{c}$; i.e., energy propagates along the characteristics which emanate from the antenna. This orthogonality of the phase and group velocities is shown in Fig. 1 of Ref. 12.

Now consider cylindrical coordinates; here, Eq. (14) becomes

$$
\mu_{1}\left[\frac{1}{r} \frac{\partial}{\partial r}\left(r \frac{\partial \varphi}{\partial r}\right)+\frac{1}{r^{2}} \frac{\partial^{2} \varphi}{\partial \theta^{2}}\right]+\frac{\partial^{2} \varphi}{\partial z^{2}}=0 .
$$

The behavior of Eq. (14) can best be appreciated when the antenna consists of a magnetic "monopole" of magnitude $q_{m}$ located at the origin, in which case Eq. (14) becomes

$\mu_{1}\left[\frac{1}{r} \frac{\partial}{\partial r}\left(r \frac{\partial \varphi}{\partial r}\right)+\frac{1}{r^{2}} \frac{\partial^{2} \varphi}{\partial \theta^{2}}\right]+\frac{\partial^{2} \varphi}{\partial z^{2}}=-4 \pi q_{m} \delta(\mathbf{r})$.

(The monopole is, of course, nonphysical but may be used as Green's function for realistic sources such as dipoles.) Equation (15) is just Poisson's equation with rescaled coordinates so that the solution, taking into acount this rescaling is

$$
\varphi(r, z)=-\frac{q_{m}}{\mu_{1}\left(z^{2}+r^{2} / \mu_{1}\right)^{1 / 2}} .
$$

When $\mu_{1}>0$ the equipotentials are simply ellipsoids, but when $\mu_{1}<0$, the equipotentials become hyperboloids with asymptotes on the cone $z= \pm r / \sqrt{-\mu_{1}}$ (note that the cone angle is just $\theta_{c}$ ). Furthermore, $\varphi$ diverges on this asymptote, so that a singularity exists on the conical surface shown in Fig. 1. In plasma physics these singular surfaces are called

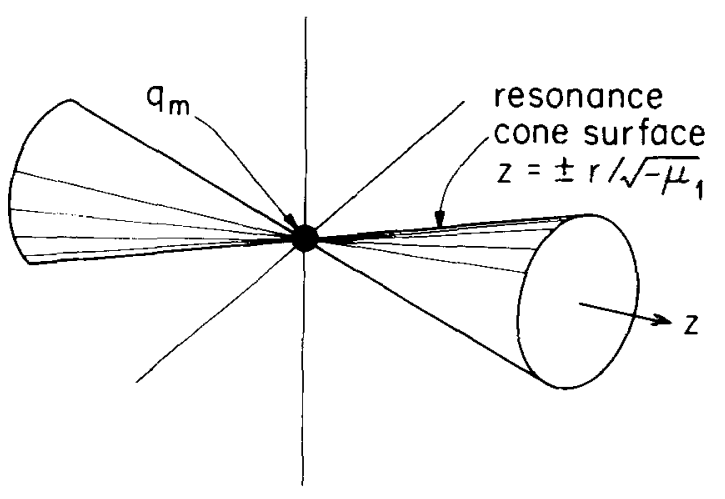

FIG. 1. Cylindrical geometry: antenna and resonance cone characteristics. resonance cones. ${ }^{2-4}$ The singularity comes about because the infinity of the potential at the point source propagates along the characteristics emanating from the source. The existence of resonance cones is not intuitively evident upon casual examination of dispersion relations like Eq. (10); to obtain resonance cones from the dispersion relation it is necessary ${ }^{2-4}$ to make a superposition of all the Fourier modes excited by a point source. These modes, each of which is infinite is spatial extent, cancel out everywhere, except on the cone.

As in plasma physics, higher order effects-which are ignored in the approximations leading to Eqs. (1) and (6)must be invoked to make the potential finite on the cone surface. These higher order effects come in as a term $\sim \epsilon \partial^{4} /$ $\partial x_{\perp}^{4}$ which is added to Eqs. (7) and (14); for plasmas $\epsilon$ depends on temperature, ${ }^{2,13}$ while for ferrites, $\epsilon$ depends on exchange effects. $^{14,15}$

Resonance cones will exist whenever $\mu_{1}<0$, which from the definition of $\mu_{1}$ occurs over the frequency band

$$
1<F^{2}<1+G \text {. }
$$

If the medium is finite rather than infinite the resonance cones can reflect off the boundaries and if the geometry is such that these reflections superimpose, then a standing wave resonance is set up which will be the Walker ${ }^{5}$ mode. In plasma physics, the same correspondence exists between resonance cones and Trivelpiece-Gould waves ${ }^{16}$ which are the analog of Walker modes. In order to see resonance cones in a finite sized ferrite it will be necessary to coat the ferrite with an absorber so that the cones do not reflect and so will be distinct from the Walker modes. (In plasmas this occurs more or less naturally, because the spatial attenuation of the corresponding plasma modes is generally much greater.) It should be noted that the nature of reflection of plasma and ferrite resonance cones is not completely analogous because, for a plasma, the parallel dielectric changes sign at a plasma vacuum interface parallel to the $z$ axis, whereas for a ferrite the perpendicular permeability changes sign at the corresponding ferrite-vacuum interface.

Finally, it is important to distinguish resonance cones from the bound magnetostatic waves discussed by Zeskind and Morganthaler, ${ }^{17}$ Morganthaler, ${ }^{15}$ and Stancil. ${ }^{14}$ A possibility for confusion exists because the modes of Refs. 14, 15 and 17 are also spatially localized. The difference lies in the fact that Refs. 14, 15, and 17 treat modes with no $z$ dependence, and rely on an inhomogeneity in the dc magnetic field to provide a material resonance which localizes the modes to a certain "virtual surface" $y=y(x)$, whereas, in contrast, resonance cones do have an explicit $z$ dependence, propagate in a homogeneous magnetic field, and do not depend on any localized resonant interaction with the medium.

This work was supported by National Science Foundation Grant ECS-8113533.

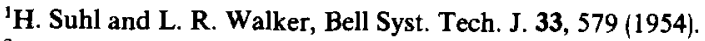

${ }^{2}$ R. K. Fisher and R. W. Gould, Phys. Rev. Lett. 22, 1093 (1969); Phys. Fluids 14, 857 (1971).

${ }^{3}$ H. H. Kuehl, Phys. Fluids 16, 1311 (1973). 
${ }^{4}$ R. J. Briggs and R. R. Parker, Phys. Rev. Lett. 29, 852 (1972).

${ }^{5}$ L. R. Walker, Phys. Rev. 105, 390 (1957).

${ }^{6} \mathrm{~L}$. K. Brundle and N. J. Freeman, Electron. Lett. 4, 132 (1968).

${ }^{7}$ M. Masuda, N. S. Chang, and Y. Matsuo, IEEE Trans. Microwave Theory Tech. MTT-19, 834 (1971).

${ }^{8}$ N. C. Srivastava, J. Appl. Phys. 47, 5447 (1976).

${ }^{9}$ Y. Ikuzawa and K. Abe, J. Appl. Phys. 48, 3001 (1977).
${ }^{11 B}$ A. Auld, J. Appl. Phys. 31, 1642 (1960).

${ }^{12}$ P. M. Bellan and M. Porkolab, Phys. Rev. Lett. 34, 124 (1975).

${ }^{13}$ P. M. Bellan, Phys. Rev. Lett. 45, 1407 (1980).

${ }^{14}$ D. D. Stancil, IEEE Trans. Magn. MAG-16, 1153 (1980).

${ }^{15}$ F. R. Morganthaler IEEE Trans. Magn. MAG-14, 806 (1978).

${ }^{16}$ A. W. Trivelpiece and R. W. Gould, J. Appl. Phys. 11, 1784 (1959).

${ }^{17}$ D. A. Zeskin and F. R. Morganthaler IEEE Trans. Magn. MAG-13, 1249 (1977). 